\title{
Respiratory evaluation of patients requiring ventilator support due to acute respiratory failure
}

\author{
Carmen Silvia Valente Barbas, Giovana Caroline Lopes, Débora Feijó Vieira, Lara Poletto Couto, \\ Letícia Kawano Dourado, Eliana Caser
}

University of São Paulo, São Paulo, Brazil

Email: carmen.barbas@gmail.com, valentebarbaspneumo@gmail.com

Received 16 September 2012; revised 19 October 2012; accepted 28 October 2012

\section{ABSTRACT}

This review, based on relevant published evidence and the authors' clinical experience, presents how to evaluate a patient with acute respiratory failure requiring ventilatory support. This patient must be carefully evaluated by nurses, physiotherapists, respiratory care practitioners and physicians regarding the elucidation of the cause of the acute episode of respiratory failure by means of physical examination with the measurement of respiratory parameters and assessment of arterial blood gases analysis to make a correct respiratory diagnosis. After the initial evaluation, the patient must quickly receive adequate oxygen and ventilatory support that has to be carefully monitored until its discontinuation. When available, a noninvasive ventilation trial must be done in patients presenting desaturation during oxygen mask and or $\mathrm{PaCO}_{2}$ retention, especially in cases of cardiogenic pulmonary edema and severe exacerbation of chronic obstructive pulmonary disease. In cases of noninvasive ventilation trial-failure, endotracheal intubation and invasive protective mechanical ventilation must be promptly initiated. In severe ARDS patients, low tidal ventilation, higher PEEP levels, prone positioning and recruitment maneuvers with adequate PEEP titration should be used. Recently, new modes of ventilation should allow a better patient-ventilator interaction or synchrony permitting a sufficient unloading of respiratory muscles and increase patient comfort. Patients with chronic obstructive pulmonary disease may be considered for a trial for early extubation to noninvasive positive pressure ventilation in centers with extensive experience in noninvasive positive pressure ventilation.

Keywords: Respiratory Failure; Noninvasive Ventilation; Endotracheal Intubation; Invasive Mechanical

Ventilation; Patient-Ventilator Synchrony

\section{INTRODUCTION}

Acute respiratory failure is a condition in which the respiratory system fails to keep an adequate arterial blood oxygenation or carbon dioxide concentration. The main causes that lead to an acute insufficient oxygenation (less than $60 \mathrm{mmHg}$ while breathing room air) or a $\mathrm{PaCO}_{2}$ elevation (more than $50 \mathrm{mmHg}$ with a concomitant decrease in arterial $\mathrm{pH}$ ) are: 1) Pulmonary parenchyma disease; 2) Cardiovascular disease (left ventricular failure, pulmonary embolism); 3) Airway disease, 4) Neuro-muscular disease; and 5) Respiratory drive alterations. It is crucial to assess the cause of the acute respiratory failure episode in order to give the patient the best treatment option allowing its reversion [1].

\section{FIRST ACTIONS IN RESPIRATORY FAILURE PATIENTS}

During the acute episode of respiratory failure, it is necessary to verify the level of consciousness, respiratory frequency, tidal volume and peripheral oxygen saturation $\left(\mathrm{SpO}_{2}\right)$ and $\mathrm{PaCO}_{2}$ levels. If the patient is hypoxemic an oxygen mask must be coupled to his/her face and pulse oximetry should be monitored to obtain a peripheral oxygen saturation above $90 \%$. Use of high flow nasal cannula oxygen (HFNC) is increasingly popular in adult ICUs for patients with acute hypoxemic respiratory failure. This is the result of the successful long-term use of HFNC in the neonatal field and recent clinical data in adults indicating beneficial effects of HFNC over conventional facemask oxygen therapy. HFNC rapidly alleviates symptoms of respiratory distress and improves oxygenation by several mechanisms, including deadspace washout, reduction in oxygen dilution and in inspiratory nasopharyngeal resistance, a moderate positive airway pressure effect that may generate alveolar recruitment and an overall greater tolerance and comfort with the interface and the heated and humidified inspired gases. Indications of HFNC are broad, encompassing most if not all causes of acute hypoxemic respiratory 
failure [2].

\section{WHEN TO PROCEED TO ENDOTRACHEAL INTUBATION? IN WHICH PATIENTS IS A NONINVASIVE VENTILATION TRIAL POSSIBLE?}

If the patient is obtunded with irregular respirations, a bag-mask ventilation with a as high as possible dose of oxygen must be coupled to the patient's face and an endotracheal intubation and mechanical ventilation must be initiated. Respiratory arrest, mental deterioration, and progressive exhaustion of respiratory muscles are also indications for endotracheal intubation

In patients already coupled to an oxygen mask that present desaturation or have an arterial $\mathrm{CO}_{2}$ above 50 mmHg with a concomitant decrease in arterial $\mathrm{pH}$ a trial of noninvasive ventilation may be initiated [3-5]. Patients with acute respiratory distress syndrome or hypoxemia, either in the postoperative setting or in the presence of immunosuppression, may also be considered for a trial of noninvasive positive pressure ventilation. A comfort interface with a shape and size that adequately fits the patient's face avoiding air-leaks is crucial for the noninvasive ventilation trial success determining the patient's compliance and the best synchrony between the patient and the machine. A variety of interfaces for noninvasive ventilation can be used in the acute care setting. Nevertheless, prevention and monitoring of interfaces related side-effects and evaluation of patient's tolerance are crucial to avoid noninvasive ventilation failure [3,4].

Assessing the success/failure of the noninvasive ventilation trial:

After 30 minutes to 2 hours of the noninvasive ventilation trial initiation the patients must present:

1) A better level of consciousness and airway protection;

2) Being comfortable with the mask;

3) Decreased/normalization of respiratory rate;

4) Increased tidal volume compared to the beginning of the trial;

5) Improved $\mathrm{PaO}_{2}$;

6) Lower $\mathrm{PaCO}_{2}$;

7) Hemodynamic stability

8) Not having a large abdominal distension.

If the patient responds well to the noninvasive mechanical ventilation trial, this ventilatory support modality must be continued while clinical treatment is concomitantly administered until the patient recovers from the acute episode of respiratory failure allowing the discontinuation of noninvasive ventilation and the reinstitution of oxygen mask or room air ventilation [5].

If the patient does not respond well to the noninvasive mechanical ventilation trial, keeping low tidal volume ventilation, high respiratory rate, limited oxygen saturation (less than $92 \%$ with $\mathrm{FIO}_{2}$ higher than $50 \%$ ), $\mathrm{pH}$ less than 7.2 and $\mathrm{PaCO}_{2}$ higher than $60 \mathrm{mmHg}$, endotracheal intubation and invasive mechanical ventilation must be promptly instituted in order to avoid complications and guarantee a secure intubation [6-8]. After intubation, the adequate positioning of the endotracheal tube must be checked with a capnography or Chest-X-ray and the tube cuff-pressure checked with a manometer and kept around 20 - 25 mmHg.

A capnography is usually used in Intensive Care to monitor ventilation, mainly in neurologic patients. Carbon dioxide levels are frequently monitored during cardiopulmonary resuscitation to aid in determining the proper placement of an endotracheal tube and to assess the effectiveness of resuscitation efforts. Although pulse oximetry is useful in the assessment of oxygenation, capnography provides more direct information on the ventilatory status of a patient. This is particularly true when patients are receiving supplemental oxygen, during which oxygen saturation may be normal despite the presence of marked hypoventilation. Conversely, capnography does not monitor oxygenation; hypoxemia may be present even when a capnography tracing is normal. There are two types of capnographs. Mainstream capnographs use sensors that are placed directly into the breathing circuit of a ventilator. Sidestream capnographs draw a sample of gas away from the breathing circuit to a separate gas sensor (The response of a sidestream device to changes in carbon dioxide concentration is delayed by a few seconds, since the gas must travel through the sampling line before it can be analyzed. However, sidestream capnographs can be used conveniently in a patient who is not intubated if the patient is fitted with a face mask or nasal cannula to monitor respiration. Nasal cannulas and face masks that feature integrated carbon dioxide sampling lines are commercially available. You can modify standard face masks by attaching the sampling line directly to the orifice of a mask or by securing the sampling line inside the mask. A normal capnogram shows a regular, nearly square waveform that oscillates at the same frequency as the patient's respiratory rate. During inspiration, the capnogram should be at zero as the patient breathes in fresh gas. When the patient starts to exhale, the first gas exhaled will be from the anatomical dead space and will contain little or no carbon dioxide. However, the concentration of carbon dioxide in the exhaled gas will rise rapidly and plateau as the alveoli begin to empty. As exhalation proceeds, the concentration of carbon dioxide remains high and increases slightly. The peak concentration reached at the end of exhalation is the $\mathrm{ETCO}_{2}$. As the patient begins to inhale again, the capnogram falls rapidly to zero, indicating the absence of carbon dioxide in the inspired gas. Capno- 
grams must always be interpreted in conjunction with other physiological variables, and clinical judgment is important. Loss of the capnographic waveform may occur in several circumstances. These include apnea, the disconnection of a ventilator circuit, the occlusion or dislodgment of an endotracheal tube, or the occlusion or disconnection of the sampling catheter. Waveforms that do not return to zero during inspiration indicate rebreathing of carbon dioxide, which can occur if the carbon dioxide absorber in an anesthesia machine is chemically exhausted, if a valve in the ventilator circuit is not functioning properly, or if the flow of fresh gas is insufficient. An elevated baseline can also be seen if the device is calibrated incorrectly. Decreases in $\mathrm{ETCO}_{2}$ can result from hyperventilation, pulmonary embolism, cardiac arrest, sudden hypotension, hypovolemia, hypothermia, leaks in the sampling system, or a partial airway obstruction [4]. Increases in $\mathrm{ETCO}_{2}$ may be caused by hypoventilation, rising body temperature, bronchospasm, adrenergic discharge, release of a tourniquet on an arm or leg, or ventilation of a previously unventilated lung. Shallow breathing causes transient lowering of $\mathrm{ETCO}_{2}$ because of dead-space ventilation, but the $\mathrm{ETCO}_{2}$ rises again after a deep inspiration when full gas exchange occurs.

A gradient exists between the $\mathrm{PaCO}_{2}$ and the $\mathrm{ETCO}_{2}$; this gradient increases as the dead-space volume increases. In disease states characterized by increased dead space and ventilation-perfusion mismatch, such as emphysema, an arterial blood gas analysis is necessary to obtain an accurate determination of the $\mathrm{PaCO}_{2}$ [9].

Several recent studies have advanced our understanding of dead-space ventilation in patients with acute lung injury/acute respiratory distress syndrome (ALI/ARDS). They have demonstrated the utility of measuring physiologic dead-space-to-tidal volume ratio (VD/VT) and related variables in assessing outcomes as well as therapeutic interventions. These studies have included the evaluation of mortality risk, pulmonary perfusion, as well as the effectiveness of drug therapy, prone positioning, positive end-expiratory pressure (PEEP) titration, and inspiratory pattern in improving gas exchange. In patients with ALI/ARDS managed with lung-protective ventilation a significant relationship between elevated VD/VT and increased mortality continues to be reported in both early and intermediate phases of ALI/ARDS. Some clinical evidence now supports the suggestion that elevated VD/VT in part reflects the severity of pulmonary vascular endothelial damage. Monitoring VD/VT also appears useful in assessing alveolar recruitment when titrating PEEP and may be a particularly expedient method for assessing the effectiveness of prone positioning. It also has revealed how subtle manipulations of inspiratory time and pattern can improve $\mathrm{CO}_{2}$ excretion.
Much of this has been accomplished using volumetric capnography. This allows for more sophisticated measurements of pulmonary gas exchange function including: alveolar VD/VT, the volume of $\mathrm{CO}_{2}$ excretion and the slope of the alveolar plateau which reflects ventilation: perfusion heterogeneity. Many of these measurements now can be made non-invasively which should only increase the research and clinical utility of volumetric capnography in studying and managing patients with ALI/ ARDS [10].

\section{USING INVASIVE MECHANICAL VENTILATION DURING ACUTE RESPIRATORY FAILURE}

Assist-controlled modes are often used after sedation and sometimes curarization for endotracheal intubation. Usually a $\mathrm{FIO}_{2}$ of $100 \%$ is set to allow an adequate oxygenation until an arterial blood gas analysis is obtained. A respiratory rate from 12 to 20 breaths per minute is often adequate in most cases, preferably being lower in obstructive patients and higher in restrictive ones. Tidal volume must be set between $5-7 \mathrm{~mL} /$ predicted body weight, the lower values being the most suited in severe cases with extremely low compliances and high resistances [6-8]. To have the fatigued respiratory muscles rest in these patients, 24 - 48 hours of full ventilatory support may be needed, with sedation to suppress the patients' respiratory effort. In severe ARDS patients $\left(\mathrm{PaO}_{2} /\right.$ $\mathrm{FIO}_{2}$ less than 120), a randomized controlled trial comparing cisatracurium to placebo for 48 hours showed an improved adjusted 90-day survival rate and increased ventilator free days in the cisatracurium group without a significant increase in muscleweakness. Short-term paralysis may facilitate patient-ventilator synchrony in the setting of lung protective ventilation. Short-term paralysis would eliminate patient triggering and expiratory muscle activity. In combination, these effects may serve to limit regional overdistension and cyclic alveolar collapse. Paralysis may also act to lower metabolism and overall ventilatory demand [11].

In ARDS patients, protective lung ventilation strategies with tidal volumes equal or less than $6 \mathrm{~mL} / \mathrm{kg}$ of predicted body weight have been associated with lower mortality when compared with $12 \mathrm{~mL} / \mathrm{kg}$ of predicted body weight $[12,13]$. Use of PEEP titrated by the lower inflection point of the inspiratory pressure-volume curve has been associated with lower mortality in two randomized, prospective and randomized trial [14,15]. Recent meta-analysis suggest survival benefits of higher PEEP levels [16] and prone position in ARDS patients $\left(\mathrm{PaO}_{2} /\right.$ $\left.\mathrm{FIO}_{2}<200\right)$ [17]. Early high pressure lung recruitment maneuvers and application of sufficient PEEP levels to maintain an open lung can be used in severe cases of 
ARDS [18,19].

Controlled hypoventilation appears to improve the clinical outcome of patients who have status asthmaticus. Low tidal volume ventilation, acceptance of higher levels of $\mathrm{PaCO}_{2}$ (till $80 \mathrm{mmHg}$ ), $\mathrm{pH}$ equal or higher than 7.2 and auto-PEEP monitoring to keep it less than $15 \mathrm{~cm}$ H20 appears to decrease barotrauma occurrence [20].

In chronic obstructive pulmonary disease patients that require invasive mechanical ventilation, an equilibrium point among low tidal volume, high inspiratory flow rate and prolonged expiratory time must be reached to avoid excessive auto-PEEP or dynamic hyperinflation $[19,20]$.

After 24 - 48 hours of respiratory muscles rest, assisted or pressure support ventilation must be initiated in order to preserve respiratory muscle activity and avoid respiratory muscle dystrophy [20,21]. Patients with chronic obstructive pulmonary disease may be considered for a trial for early extubation to noninvasive positive pressure ventilation in centers with extensive experience to decrease mechanical ventilation duration $[5,22]$.

Recently, new modes of ventilation such as neutrally adjust ventilation (NAVA), proportional assist ventilation plus (PAV-PLUS) and adaptative support ventilation (ASV) are introduced in order to increase the patient control of the ventilator and to increase information about lung mechanics and respiratory drive. These new modes of ventilation should allow a better patient-ventilator interaction or synchrony permitting a sufficient unloading of respiratory muscles and increase patient comfort [23].

\section{CONCLUSION}

The evaluation of a patient in respiratory failure is a multidisciplinary task. It should be accomplished in a timely manner, monitoring the patient, sequentially evaluating physical signs and symptoms, laboratory data and timing of noninvasive ventilation trial; endotracheal intubation. After noninvasive ventilation or mechanical ventilation is instituted, care should be taken to evaluate patient comfort, synchrony and success of ventilatory support which is a dynamic process demanding frequent reevaluations until the patient is ready to the spontaneous breathing trial (SBT) and to the extubation process.

\section{REFERENCES}

[1] McLean, B.A. (2012) Acute respiratory failure and intensive measures. Critical Care Nursing Clinics of North America, 24, 361-375. doi:10.1016/j.ccell.2012.06.008

[2] Ricard, J.D. (2012) High flow nasal oxygen in acute respiratory failure. Minerva Anestesiol, 78, 836-841.

[3] Sferrazza P.G.F., Di Marco, F., Akoumianaki, E. and Brochard, L. (2012) Recent advances in interfaces for noninvasive ventilation: From bench studies to practical issues. Minerva Anestesiol, 78, 1146-1153.
[4] Pisani, L., Carlucci, A. and Nava, S. (2012) Interfaces for noninvasive mechanical ventilation: Technical aspects and efficiency. Minerva Anestesiol, 78, 1154-1161.

[5] Keenan, S.P., Sinuff, T., Burns, K.E., Muscedere, J., Kutsogiannis, J, Mehta, S., Cook, D.J., Ayas, N., Adhikari, N.K., Hand, L., Scales, D.C., Pagnotta, R., Lazosky, L., Rocker, G., Dial, S., Laupland, K., Sanders, K., Dodek, P. and Canadian Critical Care Trials Group/Canadian Critical Care Society Noninvasive Ventilation Guidelines Group (2011) Clinical practice guidelines for the use of noninvasive positive-pressure ventilation andnoninvasive continuous positive airway pressure in the acute care setting. CMAJ, 183, E195-E214.

[6] Brochard, L., Martin, G.S., Blanch, L., Pelosi, P., Belda, F.J., Jubran, A., Gattinoni, L., Mancebo, J., Ranieri, V.M., Richard, J.C., Gommers, D., Vieillard-Baron, A., Pesenti, A., Jaber, S., Stenqvist, O. and Vincent, J.L. (2012) Clinical review: Respiratory monitoring in the ICU: A consensus of 16. Critical Care, 16, 219.

[7] Domingo, C., Blanch, L., Murias, G. and Luján, M. (2010) State-of-the-Art Sensor Technology in Spain: Invasive and non-invasive techniques for monitoring respiratory variables. Sensors (Basel), 10, 4655-4674. doi:10.3390/s100504655

[8] Kipnis, E., Ramsingh, D., Bhargava, M., Dincer, E., Cannesson, M., Broccard, A., Vallet, B., Bendjelid, K. and Thibault, R. (2012) Monitoring in the Intensive Care. Critical Care Research and Practice, 2, 473-507.

[9] Ortega, R., Connor, C., Kim, S., Djang, R. and Patel, K. (2012) Monitoring ventilation with capnography. New England Journal of Medicine, 367, e27.

[10] Kallet, R.H. (2012) Measuring dead-space in acute lung injury. Minerva Anestesiol, 78, 1297-1305.

[11] Papazian, L., Forel, J., Gacouin, A., Penot-Ragon, C., Perrin, G., Loundou, A., et al. (2010) Neuromuscular blockers in early acute respiratory distress syndrome. The New England Journal of Medicine, 363, 1107-1116. doi:10.1056/NEJMoa1005372

[12] The Acute Respiratory Distress Syndrome Network (2000) Ventilation with lower tidal volumes as compared with traditional tidal volumes for acute lung injury and the acute respiratory distress syndrome. The New England Journal of Medicine, 342, 1301e8.

[13] Burns, K.E.A., Adhikari, N.K.J., Slutsky, A.S., Guyatt, G.H., Villar, J., Zhang, H.B., Zhou, Q., Cook, D.J., Stewart, T.E. and Meade, M.O. (2011) Pressure and volume limited ventilation for the ventilatory management of patients with acute lung injury: A systematic review and meta-analysis. PLoS One, 6, e14623. doi:10.1371/journal.pone.0014623

[14] Amato, M.B., Barbas, C.S., Medeiros, D.M., Magaldi, R.B., Schettino, G.P., Lorenzi-Filho, G., Kairalla, R.A., Deheinzelin, D., Munoz, C., Oliveira, R., et al. (1998) Effect of a protective-ventilation strategy on mortality in the acute respiratory distress syndrome. The New England Journal of Medicine, 338, 347-354. doi:10.1056/NEJM199802053380602

[15] Villar, J., Kacmarek, R.M., Perez-Mendez, L. and AguirreJaime, A. (2006) A high positive end-expiratory pressure, 
low tidal volume ventilatory strategy improves outcome in persistent acute respiratory distress syndrome: A randomized, controlled trial. Critical Care Medicine, 34, 1311-1318. doi:10.1097/01.CCM.0000215598.84885.01

[16] Briel, M., Meade, M., Mercat, A., Brower, R.G., Talmor, D., Walter, S.D., Slutsky, A.S., Pullenayegum, E., Zhou, Q., Cook, D., Brochard, L., Richard, J.C., Lamontagne, F., Bhatnagar, N., Stewart, T.E. and Guyatt, G. (2010) Higher vs lower positive end-expiratory pressure in patients with acute lung injury and acute respiratory distress syndrome: Systematic review and meta-analysis. Journal of the American Medical Association, 303, 865-873. doi:10.1001/jama.2010.218

[17] Abroug, F., Ouanes-Besbes, L., Dachraoui, F., Ouanes, I. and Brochard, L. (2011). An updated study-level metaanalysis of randomised controlled trials on proning in ARDS and acute lung injury. Critical Care, 15, R6. doi:10.1186/cc9403

[18] Marini, J.J. (2010) Safer ventilation of the injured lung: One step closer. Critical Care, 14, 192. doi:10.1186/cc9028
[19] Barbas, C.S., Matos, G.F.J., Amato, M.B.P. and Carvalho, C.R.R. (2012) Goal-oriented respiratory management for critically ill patients with acute respiratory distress syndrome. Critical Care Research and Practice, 2012, 952168. doi:10.1155/2012/952168

[20] Younsuck, K. (2007) Ventilatory management in patients with chronic airflow obstruction. Critical Care Clinics, 23, 169-181.

[21] Marini, J.J. (2011) Dynamic hyperinflation and auto-positive end-expiratory pressure: Lessons learned over 30 years. American Journal of Respiratory and Critical Care Medicine, 184, 756-762.

[22] Glossop, A.J., Shepherd, N., Bryden, D.C. and Mills, G.H. (2012) Non-invasive ventilation for weaning, avoiding reintubation after extubation and in the postoperative period: A meta-analysis. British Journal of Anaesthesia, 109, 305-314. doi:10.1093/bja/aes270

[23] Moerer, O. (2012) Effort-adapted modes of assisted breathing. Current Care, 18, 61-69. 